BULL. AUSTRAL. MATH. SOC.

\title{
PERFECT MCLAIN GROUPS ARE SUPERPERFECT
}

\author{
A.J. BERRICK AND R.G. DOWNEY
}

It is shown that if a McLain group is perfect, then it is superperfect. The proof involves demonstrating that any dense linearly ordered set has the apparently stronger property of being superdense.

\section{Introduction}

The study of McLain groups $M(S, F)$ offers an attractive interplay between group theory and combinatorial set theory. This arises from the choice of a linearly ordered set $S$ in the definition. Recall (from, for example, [4], (6.2)) that this involves considering the vector space $V$ over the field $F$ whose basis elements $v_{x}$ are indexed by elements of $S . M(S, F)$ is then the group of linear transformations of $V$ into itself generated by those transformations of the form $1+a e_{x y}(a \in F$, $x<y$ in $S$ ), where $e_{x y}$ sends $v_{x}$ to $v_{y}$ and annihilates the rest of the basis. A simple example of the interaction referred to is the wellknown result (proved, for convenience, in $\$ 3$ below).

PROPOSITION 1.1. $M(S, F)$ is perfect if and only if $S$ is dense.

Here we follow standard terminology, calling a group perfect if generated by cormutators of its elements, and a set $S$ dense if whenever $x<z$ in $S$ then there exists $y$ in $(x, z)$ (that is, $x<y<z$ ).

Received 26 October 1983. The second author partially supported by singapore grant (NUS) RP-85/83.

Copyright Clearance Centre, Inc. Serial-fee code: 0004-9727/84 $\$$ A2.00 + 0.00 . 
Our aim is to provide a significant strengthening of this result, as follows. (A perfect group $M$ is said to be superperfect if every homomorphism onto $M$ having central kernel is split.)

THEOREM 1.2. If $M(S, F)$ is perfect, then it is superperfect.

The homological interpretation of this result is that (for trivial integer coefficients $) H_{1}(M(S, F))=0$ implies $H_{2}(M(S, F))=0$. This naturally leads one to ask whether it also implies that $H_{i}(M(S, F))=0$ for all $i \geq 1$. In other words:

Are perfect McLain groups acyclic?

Although we do not answer the question, this note does suggest that its answer may well lie in a deeper understanding of the nature of dense linearly ordered sets. For the proof of Theorem 1.2 given below consists first in showing that dense linearly ordered sets have an apparently stronger property. We say that $S$ is superdense if it admits a superdense fiztration $S_{0} \subset S_{1} \subset S_{2} \cdots$ (so that $S=U S_{i}$ ), that is, one for which, whenever $x<z$ in any $S_{i}$, there exists $y$ in $S_{i+1} W_{i}$ with $x<y<z$. Section 2 below is devoted to proving:

PROPOSITION 1.3. If $S$ is dense, then it is superdense.

This extra structure is employed in section 3 to give a grouptheoretic proof of the following:

PROPOSITION 1.4. If $S$ is superdense, then $M(S, F)$ is superperfect.

Our theorem is of course immediate from the three propositions. It should be remarked that the theorem can also be established without invoking the notion of superdensity [2], albeit at the expense of rather more group theory. The proof of Proposition 1.4 can be seen as grouptheoretically simpler, in much the same way as the proof of superperfectness of Steinberg groups in [1] simplifies that in [3] by exploiting the canonical filtration of the index set (the natural numbers), and so avoiding the need to establish the equivalence of all possible candidates for splittings. Also, of course, Section 2 is likely to be of interest in its own right. 


\section{Set theory}

In this section we prove Proposition 1.3. Suppose that $S$ is a dense linearly ordered set. We shall say a sequence $\left\{C_{i}\right\}$ of non-empty subsets $C_{0}, C_{1}, \ldots$ of $S$ is a d-sequence for $S$ if

(i) $C_{i} \cap C_{j}=\emptyset$ whenever $i \neq j$,

(ii) $C_{0}$ is topolagically dense in $U C_{i}$ (that is, intersects each interval $(x, y)$ of $\left.\cup C_{i}\right)$, and

(iii) given $x<y$ in any $C_{i}$, then each $C_{j}(j>i)$ intersects $(x, y)$.

Consider the collection $C$ of $d$-sequences. It is non-void, because the density of $S$ ensures that, up to order-isomorphism (and all countable linearly ordered sets without endpoints are, after Cantor, orderisomorphic), a copy of the rationals $Q$ may be embedded in $S$. Then a $d$-sequence $\left\{Q_{i}\right\}$ is defined inductively by setting $Q_{0}=\mathbb{Z}\left[2^{-1}\right]$, and, for $j \geq 1, Q_{j}=\mathbb{Z}\left[2^{-1}, 3^{-1}, 5^{-1}, \ldots, p_{j+1}^{-1}\right] \vee_{j-1}$, where $p_{k}$ denotes the $k$ th prime number. (Thus $\mathbb{Z}\left[2^{-1}, 3^{-1}, \ldots, p_{k}^{-1}\right]$ comprises those rationals that may be expressed as finite sums of the form $q=\sum_{i \leq k}\left(\sum_{n} a_{n, i} p_{i}^{-1}\right)$ with each $a_{n, i}$ an integer. So $\left.\cup Q_{i}=Q.\right)$

Now partially order $\mathcal{C}$ by defining $\left\{C_{i}\right\} \leq\left\{C_{i}^{\prime}\right\}$ if $C_{i} \leq C_{i}^{\prime}$ for all $i=0,1,2, \ldots$. Given any chain $\left\{c_{i}^{\delta}\right\}_{\delta \in \Delta}$, define $\left\{c_{i}^{\Delta}\right\}$ by $c_{i}^{\Delta}=\underset{\delta \in \Delta}{U} c_{i}^{\delta}$. Then the $c_{i}^{\Delta}$ 's are pairwise disjoint, for otherwise $x \in C_{i}^{\Delta} \cap C_{j}^{\Delta}$ implies that $x \in C_{i}^{\delta} \cap C_{j}^{\delta}$ for some $\delta \in \Delta$. Similarly we may verify that properties (ii) and (iii) hold for $\left\{c_{i}\right\}$. Hence each chain has an upper bound.

An application of Zorn's lemma now yields a maximal d-sequence $\left\{M_{i}\right\}$. We claim that $u M_{i}=S$. To prove this, we distinguish two cases. 
CASE 1. Some interval $(a, b)$ of $S$ is disjoint from $M=U M_{i}$.

In this event, as $(a, b)$ is dense, we again embed $Q$ in $(a, b)$. As before, let $\left\{Q_{i}\right\}$ be a $d$-sequence for $Q$, and consider $\left\{M_{i} \cup Q_{i}\right\}$. This sequence clearly satisfies condition (i), leaving (ii) and (iii) to be checked in order to demonstrate that it is a d-sequence. First, $M_{0} \cup Q_{0}$ is dense in $U\left(M_{i} \cup Q_{i}\right)$, for suppose $w \in M$ and $z \in \cup Q_{i}$ with $w<z$. Then there exists $x \in \cup_{i}$ such that $x<z$. However, since ( $a, b) \cap M=\emptyset$, this implies that $w<a<x<z$. From the fact that $\left\{Q_{i}\right\}$ is a $d$-sequence, there exists $y \in(x, z) \cap Q_{0}$, giving $w<a<x<y<z$. This clinches (ii). Similar reasoning accounts for (iii). Thus $\left\{M_{i} \cup Q_{i}\right\}$ is indeed a $d$-sequence, contradicting the maximality of $\left\{M_{i}\right\}$. Hence this case cannot occur.

CASE 2. Each interval of $S$ intersects $M$.

Here $M$ is topologically dense in $S$. As $M_{0}$ is topologically dense in $M$, it must therefore also be topologically dense in $S$. Take any element $a \in S \backslash \underset{i \geq 1}{\cup} M_{i}$. Define a new sequence $\left\{M_{i}^{\prime}\right\}$ via $M_{0}^{\prime}=M_{0} \cup\{a\}$ and $M_{i}^{\prime}=M_{i}$ for $i \geq 1$. Again we assert that this is a d-sequence. Only (iii) needs to be checked. Therefore suppose $x \in M_{0}$ with $a<x$, say. Because $M_{0}$ is topologically dense in $S$, we may find $y \in(x, a) \cap M_{0}$. From the fact that $\left\{M_{i}\right\}$ is a $d$-sequence, for any $j \geq 1, M_{j}^{\prime}=M_{j}$ intersects $(x, y)$ and thereby $(x, a)$. So $\left\{M_{i}^{\prime}\right\}$ is a d-sequence too. Maximality of $\left\{M_{i}\right\}$ now ensures that each $M_{i}^{\prime}=M_{i}$; in particular $a \in M_{0}$. So $M=S$, as earlier claimed.

Now define (for $i=0,1, \ldots$ ) $s_{i}=\bigcup_{j=0}^{i} M_{j}$. Then $S_{0} \subset S_{1} \subset \ldots$ is evidently a superdense filtration of $S$.

The proof above may be adapted to show that if $S$ is dense, then $S=U T_{i}$ where the $T_{i}$ 's are topologically dense, pairwise disjoint suborderings of $S$. (In particular, for $S=Q$, our given $d$-sequence has 
this property.) On the other hand, dense $S$ may have a superdense filtration $S_{0} \subset S_{1} \subset \ldots$ with no $S_{i}$ topologically dense in $S$. (For example, given any non-trivial linearly ordered set $L$, consider $S=\underset{N}{\oplus} L$ (sumned finitely and ordered lexicographically), and define $S_{i}$ to comprise those elements of $S$ that are everywhere zero after the $(i+1)$ st coordinate. In particular, this applies to $S=Q$ by choice of $L=Q$ again.) It is an open question whether such a filtration is possible for arbitrary dense $S$.

\section{Group theory}

We first establish Proposition 1.1. Both for this and subsequent arguments it is helpful to have a presentation for $M(S, F)$. We already have $\left\{1+a e_{x y} \mid a \in F, x<y\right.$ in $\left.S\right\}$ as a generating set. Moreover the composition laws $e_{x y} e_{y z}=e_{x z}$ and $e_{x y z t} e_{z t}=0$ if $y \neq z$ readily lead to relations (for arbitrary $a, b \in F$ and $x, y, z, t \in S$ )

$$
\begin{aligned}
& \left(1+a e_{x y}\right)\left(1+b e_{x y}\right)=1+(a+b) e_{x y}, \\
& {\left[1+a e_{x y}, 1+b e_{y z}\right]=1+a b e_{x z},}
\end{aligned}
$$

and

$$
\left[1+a e_{x y}, 1+b e_{z t}\right]=1 \text { if } x \neq t \text { and } y \neq z
$$

(Here $[g, h]$ is the commutator $g h g^{-1} h^{-1}$. )

To decide whether or not any other relation is a consequence of these, consider any finite product of the generators which is not made trivial by (3.1)-(3.3). The given relations enable such a product to be rewritten in the form

$$
\left(1+a e_{x_{0} y_{0}}\right) \prod_{x>x_{0}}\left(1+b_{x} e_{x y_{0}}\right) \prod_{\substack{x \\ y<y_{0}}}\left(1+c x_{x y} e_{x y}\right)
$$

for suitable $x_{0}<y_{0}$ in $S$ and non-zero $a$ in $F$. However, as a product of transformations this expression sends the basis element $v_{x_{0}}$ to 
some linear combination which includes $v_{y_{0}}$ with coefficient $a$, and is therefore non-trivial. This means

LEMMA 3.4. $M(S, F)$ is generated by alz $1+a e_{x y}$ with $a \in F$, $x<y$ in $S$, subject only to the relations (3.1)-(3.3).

It follows immediately from (3.2) that $M=M(S, F)$ is perfect whenever $S$ is dense. On the other hand, if $S$ is not dense, then let $\left(x_{0}, y_{0}\right)$ be empty in $S$. Map $M$ to the additive group of $F$ by sending $1+a e_{x_{0} y_{0}}$ to $a$, and all other generators $1+a e_{x y}$ to $0 \in F$. In view of (3.1)-(3.3) this defines a non-trivial homomorphism from $M$ to an abelian group; thus no element $1+a e_{x_{0} y_{0}}$ can be a product of

commutators and $M$ cannot be perfect. This clinches Proposition 1.1 .

For consideration of Proposition 1.4 we first perform a simplification, based on the fact that the homology of a group is the direct limit of the homology of its finitely generated subgroups. Note that, for a fixed superdense filtration $S_{0} \subset S_{1} \subset S_{2} \subset \ldots$ on $S$, any finite subset of $S$ is contained in some subset $S^{\prime}$ of $S$ such that each $S_{i}^{\prime}=S_{i} \cap S$ is finite and $S_{0}^{\prime} \subset S_{1}^{\prime} \subset S_{2}^{\prime} \subset \ldots$ is a superdense filtration on $S^{\prime}$. It therefore suffices to show that each such $M\left(S^{\prime}, E\right)$ is superperfect; accordingly, since each such $S^{\prime}$ is dense in itself, we may as well assume that our original $S$ and its superdense filtration have this "locally-finite" form.

In order to prove Proposition 1.4 we start with an arbitrary epimorphism $\phi: G \rightarrow M$ whose kernel $K$ lies in the centre $Z(G)$ of $G$. We produce a splitting $\sigma: M \rightarrow G$ (that is, $\left(1+a e_{x y}\right) \sigma \phi=1+a e_{x y}$ for all $a, x, y)$. To this end we use the superdense filtration $S_{0} \subset S_{1} \subset S_{2} \quad \ldots$ on $S$, to filter $M$ as $\underset{i \geq 0}{\cup} M_{i}$ with $M_{0} \leq M_{1} \leq \ldots$ where $M_{i}$ is the subgroup generated by all elements of the form $1+a e_{x y}, a \in F, x, y \in S_{i}$. For each $i \geq 1$ we shall define $\sigma_{i}: M_{i-1} \rightarrow G$ splitting $\phi$ over $M_{i-1}$. These splittings are compatible (that is, combine as a splitting over the inductive limit $M$ of the 
subgroups $M_{i}$ J because of the following lemma applied to the fact (obvious from (3.2)) that $M_{i-1} \leq\left[M_{i}, M_{i}\right]$.

LEMMA 3.5. Let $1:[H, H] \rightarrow H$ and $\kappa: J \rightarrow J / Z(J)$ denote the obvious inclusion and projection homomorphisms. If $\alpha, \beta: H \rightarrow J$ have $\alpha K=\beta K$, then $1 \alpha=1 \beta$.

This lemma is a consequence of the observation that, because $\alpha$ and $\beta$ agree modulo $Z(J)$, then for any $h, h^{\prime} \in H$,

$$
\left[(h) \alpha,\left(h^{\prime}\right) \alpha\right]=\left[(h) \beta Z(J),\left(h^{\prime}\right) \beta Z(J)\right]=\left[(h) \beta,\left(h^{\prime}\right) \beta\right] .
$$

Our task, once $\sigma_{i}$ has been defined, is to show that it is a homomorphism, in other words, respects the relations (3.1)-(3.3). For the definition first, for each $x$ in $S_{i-1}$ choose an element $y$ of $S_{i} V_{i-1}$ such that $x<y<z$ whenever $x<z$ in $S_{i-1}$ (possible, since the superdense filtration is also locally finite). Then, with $g_{w t}^{a}$ denoting the $K$-coset $\left(1+a e_{w t}\right) \phi^{-1}$ in $G$, let

$$
\left(1+a e_{x z}\right) \sigma_{i}=\left[g_{x y}^{1}, g_{y z}^{a}\right]
$$

By virtue of the centrality of $K$, the right-hand expression determines a single element of $G$. In particular, because $g_{y z}^{0}=K$, so

$$
(1) \sigma=\left[g_{x y}^{1}, g_{y z}^{0}\right]=1 \text {. }
$$

Next, recall the following facts from $[1, \mathrm{p} .68]$.

In any group $G$, for $u, v, w \in G$,

$$
[u, v], w]
$$

(a)

Here (a) is obtained by straightforward multiplication, and (b) by substitution for $[v, w u]=[v, 2 a v]$ in (a). Let us use (c) to prove that $\sigma$ respects $(3.3)$, by noting that, for $s, t, u, v \in S_{i}$, 


$$
\left[g_{s t}^{a}, g_{u v}^{b}\right]=1 \text { if } s \neq v \text { and } t \neq u \text {. }
$$

To see this, take $w \in S_{i+1} W_{i}$ with $s<w<t$ and observe that $g_{s t}^{a}=\left[g_{s w}^{1}, g_{w t}^{a}\right] K$. Next, by using (a) (after rearrangement) and (d) in turn, we have that

(e)

$$
\begin{aligned}
\left(1+a e_{x z}\right) \sigma_{i}\left(1+b e_{x z}\right) \sigma_{i} & =\left[g_{x y}^{1}, g_{y z}^{a}, g_{y z}^{b}\right]\left[g_{y z}^{a},\left[g_{x y}^{1}, g_{y z}^{b}\right]^{-1}\right] \\
& =\left[g_{x y}^{1}, g_{y z}^{a+b}\right]\left[g_{y z}^{a}, g_{x z}^{-b}\right. \\
& =\left(1+(a+b) e_{x z}\right) \sigma_{i} .
\end{aligned}
$$

Thus $\sigma_{i}$ also respect relations of type (3.1). Lastly, for (3.2) we apply (b), then (d), then (e), as follows:

$$
\begin{aligned}
{\left[\left(1+a e_{x z}\right) \sigma_{i},\left(1+b e_{z t}\right) \sigma_{i}\right] } & =\left[\left[g_{x y}^{1}, g_{y z}^{a}\right], g_{z t}^{b}\right] \\
& =\left[\left[g_{x y}^{1}, g_{y z}^{a}\right],\left[g_{z t}^{b}, g_{y z}^{a}\right]\right]\left[\left[g_{z t}^{b}, g_{y z}^{a}\right], g_{x y}^{1}\right] \\
& =\left[g_{x z}^{a}, g_{y t}^{-a b}\right]\left[g_{x y}^{1}, g_{y t}^{-a b}\right]^{-1} \\
& =\left(\left(1-a b e_{x t}\right) \sigma_{i}\right)^{-1} \\
& =\left(1+a b e_{x t}\right) \sigma_{i} .
\end{aligned}
$$

Thus $\sigma$ is a homomorphism after all, completing the proof of Proposition 1.4 and hence Theorem 1.2 .

\section{References}

[1] A.J. Berrick, An approach to algebraic $K$-theory (Pitman Research Notes in Mathematics, 56. Pitman, London, 1982).

[2] A.J. Berrick, "Group extensions and their trivialisation", submitted.

[3] John Milnor, Introduction to algebraic $K$-theory (Annals of Mathematical Studies, 72. Princeton University Press, Princeton, 1971). 
[4] Derek J.S. Robinson, Finiteness conditions and generalized soluble groups, Part II (Ergebnisse der Mathematik und ihrer Grenzgebiete, 63. Springer-Verlag, Berlin, Heidelberg, New York, 1972).

Department of Mathematics,

National University of Singapore,

Kent Ridge 05II,

Singapore. 